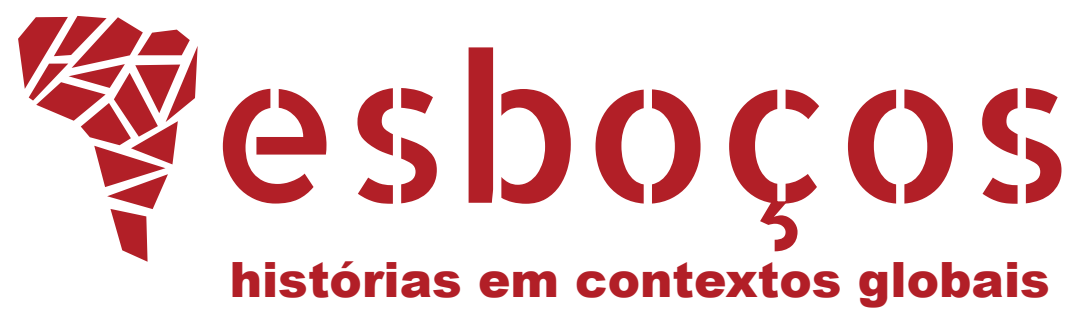

\title{
LISTA ALFABÉTICA DOS \\ PARECERISTAS QUE COLABORARAM \\ COM A ESBOÇOS: HISTÓRIAS EM \\ CONTEXTOS GLOBAIS EM 2019: \\ VOLUME 26, NÚMEROS 41, 42 E 43
}

Alphabetical list of reviewers who contributed to Esboços: histories in

global contexts in 2019: Volume 26, No. 41, 42, and 43

Flávia Florentino Varella $\mathrm{b}$

(D) https://orcid.org/0000-0001-7123-8807

E-mail: flavia_varella@hotmail.com

Bruna Vitória Grandocd

(D) https://orcid.org/0000-0002-5142-0131

E-mail: brugrando2010@hotmail.com

ac Universidade Federal de Santa Catarina, Centro de Filosofia e Ciências Humanas, Departamento de História, Florianópolis, SC, Brasil

${ }^{\mathrm{b}}$ Editora-chefe da Esboços: histórias em contextos globais

d Estagiária da Esboços: histórias em contextos globais 
Alexandre Busko Valim, Universidade Federal de Santa Catarina, Florianópolis, SC, Brasil

Ana Carolina Schveitzer, Humboldt Universität zu Berlin, Berlim, Alemanha

Ana Flavia Cernic Ramos, Universidade Federal de Uberlândia, Uberlândia, MG, Brasil

André de Lemos Freixo, Universidade Federal de Ouro Preto, Mariana, MG, Brasil

Antonio Luigi Negro, Universidade Federal da Bahia, Salvador, BA, Brasil

Arthur Curvelo, Universidade de Lisboa, Lisboa, Portugal

Beatriz Mamigonian, Universidade Federal de Santa Catarina, Florianópolis, SC, Brasil

Bruno Ayllon, Universidad Complutense de Madrid, Madri, Espanha

Carlos Augusto Machado, University of St Andrews, St Andrews, Escócia

Carlos Riojas, Universidad de Guadalajara, Zapopan, JAL, México

Cláudia de Jesus Maia, Universidade Estadual de Montes Claros Montes Claros, MG, Brasil

Cláudia Regina Bovo, Universidade Federal do Triângulo Mineiro, Uberaba, MG, Brasil

Daniel da Silva Klein, Universidade Federal do Acre, Rio Branco, AC, Brasil

Daniela Queiroz Campos, Universidade Federal de Santa Catarina, Florianópolis, SC, Brasil

Denilson Botelho de Deus, Universidade Federal de São Paulo, Guarulhos, SP, Brasil

Dominique Vieira Coelho dos Santos, Universidade Regional de Blumenau, Blumenau, SC, Brasil

Êça Pereira da Silva, Universidade Federal do Tocantins, Palmas, TO, Brasil

Elaine Ribeiro da Silva dos Santos, Universidade Federal de Alfenas, Alfenas, MG, Brasil

Elenita Malta Pereira, Universidade Estadual do Centro-Oeste, Guarapuava, PR, Brasil 
Ely Bergo de Carvalho, Universidade Federal de Minas Gerais, Belo Horizonte, MG, Brasil

Erick Assis de Araújo, Universidade Estadual do Ceará, Fortaleza, CE, Brasil

Fábio Afonso Frizzo de Moraes Lima, Universidade Federal do Triângulo Mineiro, Uberaba, MG, Brasil

Fábio Duarte Joly, Universidade Federal de Ouro Preto, Mariana, MG, Brasil

Fábio Franzini, Universidade Federal de São Paulo, Garulhos, SP, Brasil

Fábio Kühn, Universidade Federal do Rio Grande do Sul, Porto Alegre, RS, Brasil

Fábio Pádua dos Santos, Universidade Regional de Blumenau, Blumenau, SC, Brasil

Fernanda Sposito, Brown University, Province, RI, Estados Unidos da América

Gabriel Aladren, Universidade Federal Fluminense, Rio de Janeiro, RJ, Brasil

Gilvan Odival Veiga Dockhorn, Universidade Federal de Santa Maria, Santa Maria, RS, Brasil

Glaucia Cristina Candian Fraccaro, Pontifícia Universidade Católica de Campinas, Campinas, SP, Brasil

Gustavo Junqueira Duarte Oliveira, Pontifícia Universidade Católica de Campinas, Campinas, SP, Brasil

Henrique Espada Lima Filho, Universidade Federal de Santa Catarina, Florianópolis, SC, Brasil

Hilda Pivaro Stadniky, Universidade Estadual de Maringá, Maringá, PR, Brasil Jean-Pierre Hérubel, Purdue University, West Lafayette, IN, Estados Unidos da América

João Batista de Jesus Felix, Universidade Federal do Tocantins, Tocantinópolis, TO, Brasil

João Emiliano Fortaleza de Aquino, Universidade Estadual do Ceará, Fortaleza, CE, Brasil

João Júlio Gomes dos Santos Júnior, Universidade Estadual do Ceará, Fortaleza, CE, Brasil

José Carlos Vilardaga, Universidade Federal de São Paulo, Guarulhos, SP, Brasil 
Juliana Sayuri Ogassawara, Universidade Federal de Santa Catarina, Florianópolis, SC, Brasil

Larissa Rosa Correa, Pontifícia Universidade Católica do Rio de Janeiro, Rio de Janeiro, RJ, Brasil

Leandro Calbente Câmara, pesquisador autônomo

Leandro Duarte Rust, Universidade Federal de Mato Grosso, Cuiaba, MT, Brasil Leonardo Marques, Universidade Federal Fluminense, Niterói, RJ, Brasil

Lindener Pareto Júnior, Pontifícia Universidade Católica de Campinas, Campinas, SP, Brasil

Luis Fernando Bernardi Junqueira, Universidade de Fudan, Shanghai, China

Mafalda Soares da Cunha, Universidade de Évora, Évora, EVR, Portugal

Manoela da Silva Pedroza, Universidade Federal do Rio de Janeiro, Rio de Janeiro, RJ, Brasil

Marcelo Vianna, Instituto Federal de Educação, Ciência e Tecnologia do Rio Grande do Sul, Osório, RS, Brasil

Márcia Pereira dos Santos, Universidade Federal de Goiás, Catalão, GO, Brasil

Marco Aurélio dos Santos, Universidade de São Paulo, São Paulo, SP, Brasil

Marcos Gerhardt, Universidade de Passo Fundo, Passo Fundo, RS, Brasil

Mateus Henrique de Faria Pereira, Universidade Federal de Ouro Preto, Mariana, MG, Brasil

Miguel Angel Guzmán López, A Universidade de Guanajuato, Guanajuato, GTO, México

Monica Grin Monteiro de Barros, Universidade Federal do Rio de Janeiro, Rio de Janeiro, RJ, Brasil

Nádia Farage, Universidade Estadual de Campinas, Campinas, SP, Brasil

Nuno Fernando de Pinho e Silva de Almeida Falcão, Universidade da Integração Internacional da Lusofonia Afro-Brasileira, Redenção, CE, Brasil

Oldimar Pontes Cardoso, Instituto Brasileiro de Informação em Ciência e Tecnologia, Rio de Janeiro, RJ, Brasil 
Omar Acha, Universidad de Buenos Aires, Buenos Aires, BA, Argentina

Patricia Vieira, Georgetown University, Washington, D.C, Estados Unidos da América

Pedro Spinola Pereira Caldas, Universidade Federal do Estado do Rio de Janeiro, Rio de Janeiro, RJ, Brasil

Pedro Telles da Silveira, Universidade Federal do Rio Grande do Sul, Porto Alegre, RS, Brasil

Peter Frankopan, Oxford University, Oxford, Inglaterra

Ricardo Souza de Carvalho, Universidade de São Paulo, São Paulo, SP, Brasil

Roberto Moll, Universidade Federal Fluminense, Niterói, RJ, Brasil

Robson Murilo Grando Della Torre, Universidade Estadual de Montes Claros, São Francisco, MG, Brasil

Rodrigo Bragio Bonaldo, Universidade Federal de Santa Catarina, Florianópolis, SC, Brasil

Rodrigo Goyena da Silveira Soares, Universidade de São Paulo, São Paulo, SP, Brasil

Rodrigo Turin, Universidade Federal do Estado do Rio de Janeiro, Rio de Janeiro, RJ, Brasil

Samira Peruchi Moretto, Universidade Federal da Fronteira Sul, Chapecó, SC, Brasil

Sandra Mara Dantas, Universidade Federal do Triângulo Mineiro, Uberaba, MG, Brasil

Semíramis Corsi Silva, Universidade Federal de Santa Maria, Santa Maria, RS, Brasil

Sidnei Munhoz, Universidade Estadual de Maringá, Maringá, PR, Brasil

Thiago Nascimento Krause, Universidade Federal do Estado do Rio de Janeiro, Rio de Janeiro, RJ, Brasil

Tiago Kramer de Oliveira, Universidade Federal de Santa Catarina, Florianópolis, SC, Brasil

Uiran Gebara da Silva, Universidade Federal Rural de Pernambuco, Recife, PE, Brasil

Vitor Eduardo Schincariol, Universidade Federal do ABC, Santo André, SP, Brasil 


\section{NOTAS}

\section{AUTORIA}

Flávia Florentino Varella: Doutora. Professora adjunta, Universidade Federal de Santa Catarina, Centro de Filosofia e Ciências Humanas, Departamento de História, Florianópolis, SC, Brasil.

Bruna Vitória Grando: Graduanda, Universidade Federal de Santa Catarina, Centro de Filosofia e Ciências Humanas, Departamento de História, Florianópolis, SC, Brasil.

\section{ENDEREÇO PARA CORRESPONDÊNCIA}

Flávia Florentino Varella. Departamento de História, CFH, Bloco E, $6^{\circ}$ andar, Campus Universitário da UFSC, Trindade, 88040-970, Florianópolis, SC, Brasil.

\section{LICENÇA DE USO}

Esta lista de pareceristas está licenciada sob a Licença Creative Commons CC-BY. Com essa licença você pode compartilhar, adaptar, criar para qualquer fim, desde que atribua a autoria da obra.

\section{PUBLISHER}

Universidade Federal de Santa Catarina. Programa de Pós-Graduação em História. Portal de Periódicos UFSC. As ideias expressadas neste artigo são de responsabilidade de seus autores, não representando, necessariamente, a opinião dos editores ou da universidade.

\section{EDITORES}

Alex Degan

Beatriz Mamigonian

Fábio Augusto Morales

Flávia Florentino Varella (Editora-chefe)

Tiago Kramer de Oliveira

Waldomiro Lourenço da Silva Júnior 